\title{
Resonancia magnética de médula espinal y cerebro en el correlato clínico de la paraparesia espástica progresiva que se asocia al virus humano linfotrópico tipo-I (HTLV-I)
}

\author{
Jorge Cervilla $0^{1}$, Luis Cartier $\mathbf{R}^{2}$, Luis García $F^{3}$. \\ Brain and spinal cord magnetic \\ resonance imaging in spastic \\ paraparesis associated to human \\ T-lymphotropic virus
}

Background: The spastic paraparesis associated to HTLV-1 causes degenerative pyramidal tract lesions of the spinal cord and affects cortical-nuclear connections in the brain. Aim: To report the findings of magnetic resonance imaging in patients with spastic paraparesis. Material and methods: A magnetic resonance imaging of the brain and spinal cord was performed in 30 patients ( 24 females), mean age and evolution of 56 and 12 years respectively, with a clinical and virological diagnosis of tropical spastic paraparesis/HTLV-1 associated myelopathy (TSP/HAM). Results: No patient had abnormal signals in the spinal cord parenchyma. However, an atrophy of the dorsal segment was observed in $87 \%$ of patients. Patients with the highest degree of atrophy showed a higher degree of functional impairment. Eleven patients had spinal cord conus atrophy, associated to neurogenic bladder or impotency. In 80\% of patients, hyperintense subcortical white matter images in DP, T2 and Flair, mostly bi frontal, were detected. In half of them, small rounded and isolated images were observed. In the other half, eight or more images, generally larger and occasionally confluent, were found. Ten of 12 patients with confluent brain lesions showed different degrees of cognitive impairment. No patient had lesions in the corpus callosus, periventricular white matter, pons, medulla oblongata or cerebellum. Conclusions: Most patients with tropical spastic paraparesis have alterations in brain or spinal cord magnetic resonance imaging. The magnetic resonance lesions are concordant with functional impairment. The characteristics of the imaging in TSP/HAM patients can be helpful in the differential diagnosis of patients with paraparesis (Rev Méd Chile 2006; 134: 1011-20).

(Key words: Human T-lymphotropic virus 1; Magnetic resonance imaging; Paraparesis, tropical spastic)

Recibido el 4 de enero, 2005. Aceptado el 8 de marzo, 2006.

${ }^{1}$ Neurorradiología Diagnoimagen. ${ }^{2}$ Departamento de Ciencias Neurológicas, Facultad de Medicina, Universidad de Chile. Hospital del Salvador. ${ }^{3}$ Servicio de Neurología, Hospital Naval Almirante Nef.

Correspondencia a: Dr. Luis Cartier R.

E mail: lcartier@med.uchile,cl 
L a paraparesia espática tropical (TSP) ${ }^{1}$ o mielopatía asociada a HTLV-I (HAM) ${ }^{2}$ es una enfermedad que clínicamente se caracteriza por disminución progresiva de la potencia muscular de las extremidades inferiores, espasticidad, hiperreflexia y signo de Babinski, además de vejiga neurogénica, producto del daño en la vía motora central $^{3}$.

Los estudios histopatológicos han demostrado que en los segmentos lumbares, dorsales $\mathrm{y}$, a veces, cervicales, se observan lesiones simétricas de la vía motora (córtico-espinal), que se definen por la pérdida de axones y desmielinización ${ }^{4,5}$. Asimismo, en algunos casos se ha encontrado daño en la vía propioceptiva de las piernas (haz de Goll) en los segmentos cervicales ${ }^{5,6}$. Histológicamente, hasta $50 \%$ de los TSP/HAM se acompaña de manguitos linfocitarios perivasculares en los vasos de la médula espinal, hecho independiente de las lesiones sistematizadas del parénquima nervioso ${ }^{7,8}$. La degeneración ascendente de las fibras motoras y descendente de las fibras sensitivas, define un compromiso primario y distal de los axones largos, «degeneración axomielínica». Por otra parte, las lesiones parenquimatosas no están limitadas a la médula espinal, también se han observado lesiones en la sustancia blanca subcortical tanto en estudios histopatológicos ${ }^{5,9}$ como de resonancia magnética ${ }^{10,11}$, sugiriendo que la enfermedad abarca varias estructuras específicas del sistema nervioso central (SNC).

El propósito de este estudio es correlacionar las manifestaciones clínicas del TSP/HAM y los cambios anatómicos observables en la resonancia magnética (RM), en una pacientes portadores de HAM/TSP.

\section{Material y MÉTOdo}

Se diagnosticaron como TSP/HAM 30 pacientes, cuyos parámetros clínicos, electrofisiológicos y de laboratorio concordaban con las definiciones de la enfermedad previamente establecida ${ }^{3}$. Los pacientes son controlados por varios años en el Servicio de Neurología del Hospital del Salvador.

El HTLV-I fue definido con Elisa, inmunofluorescencia con células MT2, Western-blot y PCR con secuencias de: tax y 5'ltr. La evaluación psicométrica se hizo sobre la base del test de
Wechler y del test de retención visual de Benton $^{12}$. La funcionalidad motora se evaluó de acuerdo a la capacidad de marcha, estableciéndose 3 grupos: marcha independiente, marcha con necesidad de apoyo y sin capacidad de marcha. La evaluación funcional de la vejiga y esfínteres se realizó en una anamnesis dirigida a establecer: frecuencia miccional, presencia de nicturia, urgencia y capacidad de contención esfinteriana. En algunos pacientes, se hizo estudio urodinámico para establecer la existencia de una disinergia vesical. La disfunción eréctil también se identificó anamnésticamente.

La RM se efectuó en un equipo General Electric 1,5T Signa (Milwakee, Wi). Se practicaron secuencias T1, densidad protónica, T2 y FLAIR en el encéfalo en los tres planos espaciales. Toda la médula espinal se estudió con secuencias T1 y T2 en los planos axial y sagital. También se efectuaron secuencias T1 posinyección intravenosa de contraste paramagnético, para la evaluación del encéfalo y la médula.

En un grupo control de 30 pacientes, de similar edad, se efectuó medición de los diámetros A-P y transversal máximos de la médula espinal en el nivel D8 y también en el cono medular, para establecer valores de referencia. En base a ello, se pudo definir tres grados en la atrofia de la médula espinal: leve entre 4 y $6 \mathrm{~mm}$, moderada entre 3 y $4 \mathrm{~mm}$ y severa $3 \mathrm{~mm}$ o menos. De igual modo, se establecieron tres grados de atrofia en el cono medular: leve entre 5,0 y $6,5 \mathrm{~mm}$, moderada entre 3,5 y $5,0 \mathrm{~mm}$ y severa $3,5 \mathrm{~mm}$ o menos. Uno de los casos tiene estudio neuropatológico.

\section{RESULTADOS}

De los 30 pacientes estudiados, 24 eran mujeres y 6 hombres, que promediaban una edad de 56,1 años (26-73 años). El tiempo de evolución de su enfermedad era de 11,6 años (3-33 años). La definición de funcionalidad motora estableció que 10 pacientes tenían marcha independiente, 12 enfermos tenían marcha pareto-espástica con necesidad de apoyo y 8 no tenían marcha. Todos los pacientes, salvo 2 , tenían algún grado de vejiga neurogénica y $66,6 \%$ de los hombres padecían disfunción eréctil (Tabla 1). El estudio neuropsicológico reveló que 11 pacientes $(36,6 \%)$ presenta- 
Tabla 1. D escripción de casos estudiados

\begin{tabular}{|c|c|c|c|c|c|c|c|c|}
\hline \multirow[b]{2}{*}{ № } & \multirow[b]{2}{*}{$\begin{array}{l}\text { Edad } \\
\text { Sexo }\end{array}$} & \multirow[b]{2}{*}{$\begin{array}{l}\text { Evol. } \\
\text { Años }\end{array}$} & \multicolumn{2}{|c|}{ Médula } & \multicolumn{2}{|c|}{ Cono } & \multicolumn{2}{|c|}{ Cerebro } \\
\hline & & & $\begin{array}{c}\text { Función } \\
\text { motora }\end{array}$ & $\begin{array}{l}\text { Atrofia } \\
\text { medular }\end{array}$ & $\begin{array}{c}\text { Vejiga } \\
\text { neurogénica }\end{array}$ & Cono & $\begin{array}{l}\text { Deterioro } \\
\text { intelectual }\end{array}$ & $\begin{array}{l}\text { Lesiones } \\
\text { cerebrales }\end{array}$ \\
\hline 1 & $55 / F$ & 22 & M. con apoyo & Leve & Urgencia & Normal & Sin deterioro & Aisladas \\
\hline 2 & $42 / \mathrm{M}$ & 4 & Sin marcha & Moderada & Urgencia (i) & Atrofia & Sin deterioro & Aisladas \\
\hline 3 & $71 / \mathrm{F}$ & 9 & M. con apoyo & Severa & Urgencia & Normal & Deterioro $(+)$ & Confluentes \\
\hline 4 & $56 / F$ & 5 & M.independiente & Normal & Frecuencia & Normal & Deterioro $(+)$ & Aisladas \\
\hline 5 & $63 / \mathrm{F}$ & 9 & M. con apoyo & Moderada & Frecuencia & Atrofia & Sin deterioro & Aisladas \\
\hline 6 & $59 / F$ & 12 & M.independiente & Leve & Normal(an) & Normal & Sin deterioro & Normal \\
\hline 7 & $73 / \mathrm{F}$ & 28 & Sin marcha & Severa & Incontinencia & Atrofia & Deterioro (+) & Confluentes \\
\hline 8 & $59 / F$ & 5 & M. con apoyo & Moderada & Incontinencia (an) & Atrofia & Deteriom $(+)$ & Confluentes \\
\hline 9 & $42 / F$ & 9 & M.independiente & Normal & Urgencia (an) & Normal & Sin deterioro & Normal \\
\hline 10 & $68 / \mathrm{F}$ & 14 & Sin marcha & Moderada & Frecuencia & Normal & Sin deterioro & Normal \\
\hline 11 & $58 / \mathrm{F}$ & 12 & M. con apoyo & Severa & Urgencia & Atrofia & Deterioro $(+)$ & Confluentes \\
\hline 12 & $71 / \mathrm{F}$ & 8 & M. con apoyo & Moderada & Frecuencia & Normal & Deterioro $(+)$ & Confluentes \\
\hline 13 & $59 / \mathrm{F}$ & 7 & M.independiente & Normal & Normal & Normal & Sin deterioro & Aisladas \\
\hline 14 & $65 / F$ & 15 & M. con apoyo & Leve & Frecuencia & Normal & Sin deterioro & Aisladas \\
\hline 15 & $39 / \mathrm{M}$ & 7 & M.independiente & Moderada & Frecuencia & Normal & Deteriom $(+)$ & Confluentes \\
\hline 16 & $64 / \mathrm{M}$ & 8 & M.independiente & Leve & Frecuencia & Normal & Sin deterioro & Aisladas \\
\hline 17 & $68 / \mathrm{M}$ & 10 & M.independiente & Severa & Frecuencia (i) & Atrofia & Sin deterioro & Aisladas \\
\hline 18 & $47 / \mathrm{F}$ & 4 & M.independiente & Moderada & Frecuencia (an) & Normal & Sin deterioro & Normal \\
\hline 19 & $50 / F$ & 5 & M. con apoyo & Leve & Urgencia & Normal & Sin deterioro & Normal \\
\hline 20 & $58 / \mathrm{F}$ & 15 & Sin marcha & Moderada & Urgencia & Normal & Deteriom $(+)$ & Confluentes \\
\hline 21 & $56 / F$ & 33 & M. con apoyo & Severa & Urgencia & Atrofia & Sin deterioro & Confluentes \\
\hline 22 & $55 / F$ & 24 & Sin marcha & Severa & Incontinencia & Atrofia & Deterioro ( + ) & Confluentes \\
\hline 23 & $67 / \mathrm{F}$ & 12 & M. con apoyo & Moderada & Urgencia & Normal & Sin deterioro & Aisladas \\
\hline 24 & $59 / \mathrm{F}$ & 3 & M.con apoyo & Normal & Frecuencia & Normal & Sin deterioro & Normal \\
\hline 25 & $29 / F$ & 9 & M.independiente & Leve & Frecuencia & Normal & Sin deterioro & Aisladas \\
\hline 26 & 39/M & 18 & Sin marcha & Severa & Urgencia (i) & Atrofia & Sin deterioro & Aisladas \\
\hline 27 & $66 / F$ & 9 & M. con apoyo & Moderada & Urgencia & Atrofia & Deterioro (+) & Confluentes \\
\hline 28 & $26 / F$ & 3 & M.independiente & Moderada & Frecuencia & Normal & Sin deterioro & Confluentes \\
\hline 29 & $56 / F$ & 3 & Sin marcha & Moderada & Frecuencia & Normal & Sin deterioro & Aisladas \\
\hline 30 & $65 / \mathrm{M}$ & 26 & Sin marcha & Severa & Urgencia (i) & Atrofia & Deteriom $(+)$ & Confluentes \\
\hline
\end{tabular}

M= marcha; i= impotencia; an= ano neurogénico.

ban algún grado de déficit cognitivo en las pruebas de WAIS y Benton. Se pudo definir: compromiso de la memoria de corto plazo, disminución de la capacidad de aprendizaje, disfunciones viso-constructivas y limitaciones de la capacidad de planificación ${ }^{12}$. Seis pacientes presentaban un déficit cognitivo leve, en 5 enfermos era moderado, y en 19 casos no se demostró compromiso cognitivo, con los tests usados.

La resonancia magnética de la médula espinal no mostró señales anormales, en ninguno de los pacientes. El hallazgo más frecuente, en $86,6 \%$ de los casos, fue la atrofia de la médula espinal en el nivel dorsal. En dos pacientes (casos 21 y 22) también se observó atrofia de la médula cervical. De acuerdo a la intensidad, la atrofia dorsal fue severa en 8 pacientes; en los que la médula tendió a presentar una forma triangular de vértice posterior, o se aplanó en el sentido anteroposterior (Figuras 1 y 2). En 12 enfermos, la atrofia fue moderada y en 6 , fue leve; sólo en 4 pacientes la médula presentó diámetro normal. El diámetro 
coronal de la médula espinal no se modificó significativamente en los TSP/HAM. Además, en 11 pacientes se encontró una disminución en el volumen del cono de la médula espinal (Figura 3). Esta atrofia del cono medular fue severa en 6 pacientes, moderada en 4 y sólo leve en uno de ellos. No se pesquisaron captaciones patológicas del medio de contraste paramagnético en la médula espinal, ni en las regiones perimedulares.

Los 8 pacientes que mostraban un grado severo de atrofia medular tenían como tiempo promedio de enfermedad 20 años $( \pm 8,4$ años). El grupo de pacientes que mostraba atrofia leve o moderada de la médula, tenían un promedio de evolución de 9 años ( $\pm 3,9$ años). Los dos pacientes en los que se encontró atrofia de la médula cervical presentaban una paraparesia de larga evolución (24 y 33 años). Los 4 enfermos en los que se observó normal la médula, el tiempo de evolución era de 6,4 años, en promedio. Con esta información, se pudo definir una relación inversa entre el tiempo de evolución del TSP/HAM y el diámetro de la médula. En la Figura 4 se aprecia la médula dorsal de un caso con larga evolución de TSP/HAM comparada con una médula normal.

Entre los 20 enfermos que mostraron una atrofia severa o moderada de la médula espinal

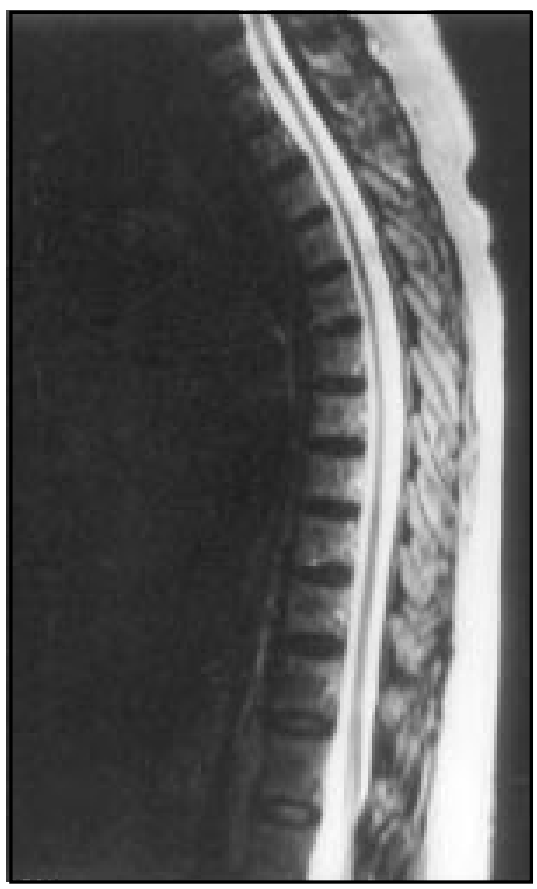

Figura 1. Imagen sagital T2 que muestra una marcada atrofia de la médula dorsal, con intensidad de señal respetada. También se evidencia importante atrofia del cono medular (caso 11).

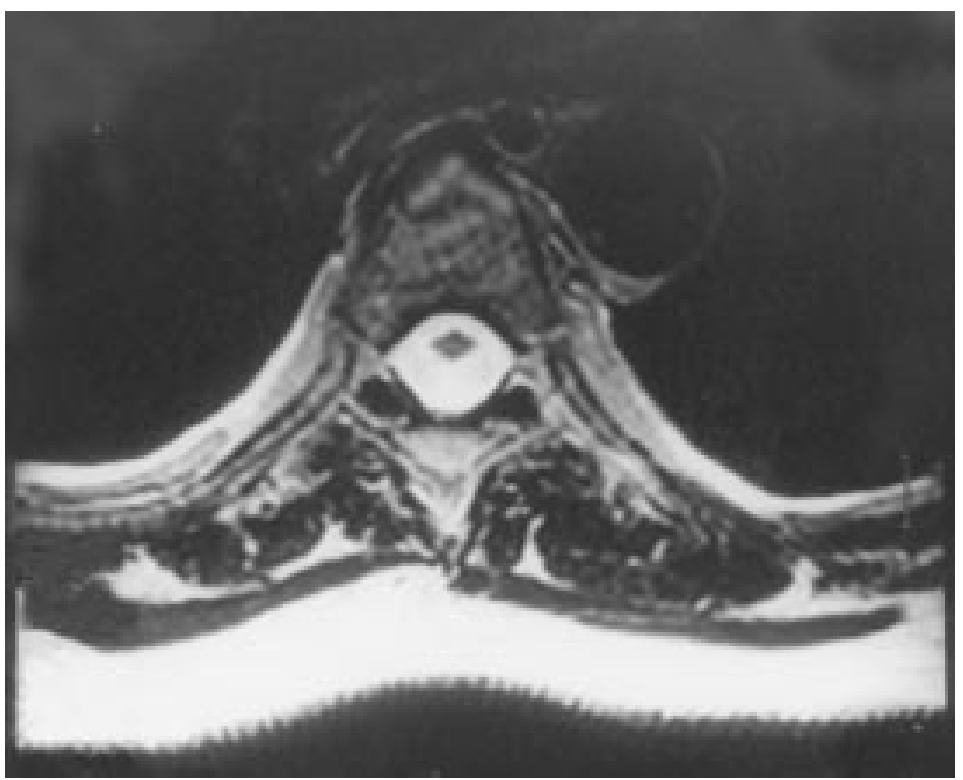

Figura 2. Imagen axial T2. Nótese importante atrofia medular que adquiere una configuración triangular de vértice posterior. La intensidad de señal está conservada (caso 7). 


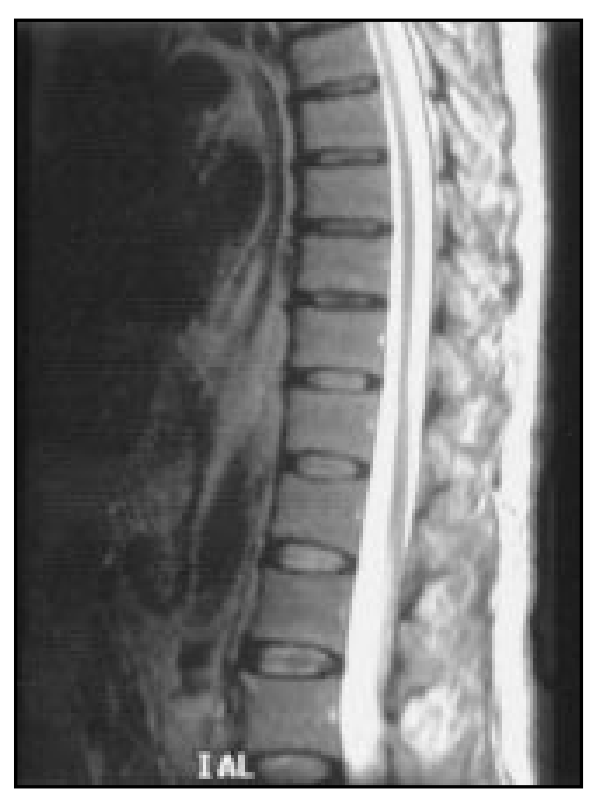

Figura 3. Imagen sagital T2. Importante atrofia difusa del cono medular, sin alteración asociada de su intensidad de señal (caso 17).

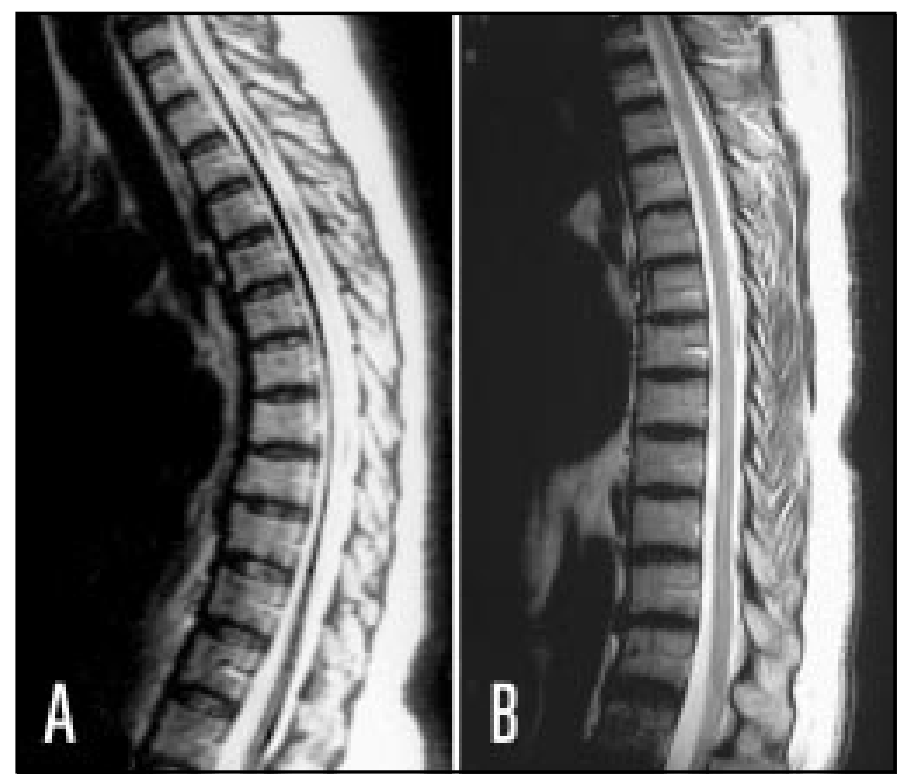

Figura 4. Imagen sagital T2 (A). Paciente portador de paraparesia de larga data que muestra una importante atrofia medular prácticamente filiforme (caso 21). (B) Compárese con una médula dorsal normal en imagen sagital T2 de un paciente control de similar edad. (menos de $4 \mathrm{~mm}$ ), se contaban los pacientes que tenían el mayor compromiso funcional, todos los enfermos que no tenían marcha y $66,6 \%$ de los pacientes con necesidad de apoyo para caminar. Por el contrario, el grupo de 10 pacientes que tenía médula normal o con leve atrofia $(5 \mathrm{~mm} 0$ más), estaba constituido por pacientes con marcha independiente y sólo por 33,3\% de los pacientes que necesitaban apoyo para caminar.

Entre los 11 enfermos que presentaron atrofia del cono medular, estaban los únicos tres pacientes con incontinencia vesical y $50 \%$ de los pacientes que sufrían de urgencia miccional. Además, estaban todos los hombres que padecían de falla eréctil. Hubo 19 pacientes en que no se observó cambios en las imágenes del cono medular. Este grupo estaba constituido por los dos enfermos sin trastornos vesicales, por 11 enfermos que sólo tenían aumento de la frecuencia miccional (trastorno que suele asociarse con vejigas espásticas) y por 6 casos que, teniendo urgencia miccional, no se expresaban con cambios estructurales. De los tres casos con ano neurogénico, sólo uno se correlacionó con atrofia del cono.
La resonancia magnética del cerebro demostró presencia de imágenes patológicas en $80,0 \%$ de los TSP/HAM estudiados. Un grupo (40\%) presentaba pequeñas lesiones hiperintensas aisladas en DP, T2 y FLAIR, habitualmente no mayores de $5 \mathrm{~mm}$ de diámetro, de forma ovalada o redondeada, distribuidas en la sustancia blanca subcortical, predominando en las regiones fronto-insulares, fronto-centrales y en áreas fronto-estriadas. Estas lesiones respetaban las fibras subcorticales en $U$, estaban bien delimitadas y no ejercían efecto de masa. Ninguna de las lesiones descritas mostró captación de gadolinio. Este patrón aislado» de pequeñas lesiones hiperintensas tendía a ser bilateral y simétrico (Figuras 5 y 6).

Un segundo grupo, formado por 12 enfermos presentaba más de 8 lesiones de mayor tamaño, de bordes menos definidos y con tendencia a ser confluentes. Comprometían primordialmente la sustancia blanca subcortical bifrontal o la región anterior de los centros semiovales, adoptando una disposición «arciforme». Estas lesiones fluctuaban entre 5 y $15 \mathrm{~mm}$ de diámetro y podrían agruparse bajo la denominación de «patrón confluente» (Figuras 7 y 8). 


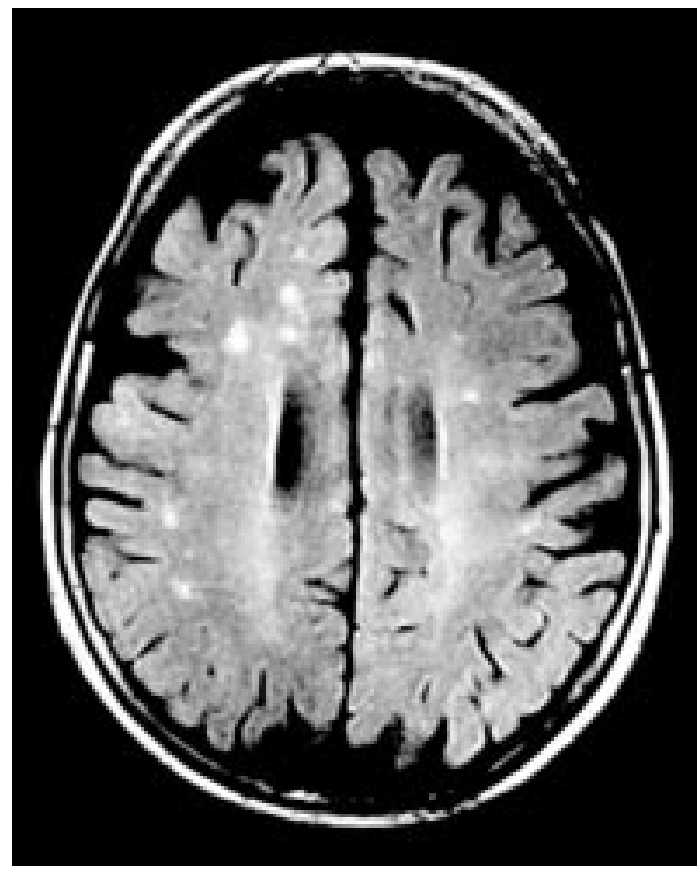

Figura 5. Imagen axial FLAIR que muestra pequeñas lesiones hiperintensas aisladas en la sustancia blanca subcortical, bien delimitadas y sin efecto de masa (caso 1).

No se encontraron lesiones en el cuerpo calloso, sustancia blanca periventricular profunda, tampoco en el tronco del encéfalo ni en el cerebelo. Del total de pacientes, sólo 6 mostraron algún grado de atrofia cerebral, de predominio cortical, que no estaba relacionada con la intensidad de las lesiones de la sustancia blanca. Este grupo de pacientes tenía en promedio 64,5 años de edad.

En la Figura 9, se reconoce histológicamente pequeñas lesiones subcorticales como las que se encontraron en el primer grupo y se observa, asimismo, las características de la médula espinal de un paciente que llegó a la anatomía patológica.

El 75\% de los enfermos con atrofia severa de la médula espinal presentaba, además, el mayor número de lesiones en la sustancia blanca subcortical. Sin embargo, 6 pacientes con lesiones confluentes en la sustancia blanca subcortical, sólo presentaban moderada atrofia medular.

Del total de pacientes que tenían lesiones confluentes cerebrales, diez de ellos (76,9\%) mostraba algún grado de deterioro cognitivo. Sólo tres casos que presentaban lesiones confluentes en la

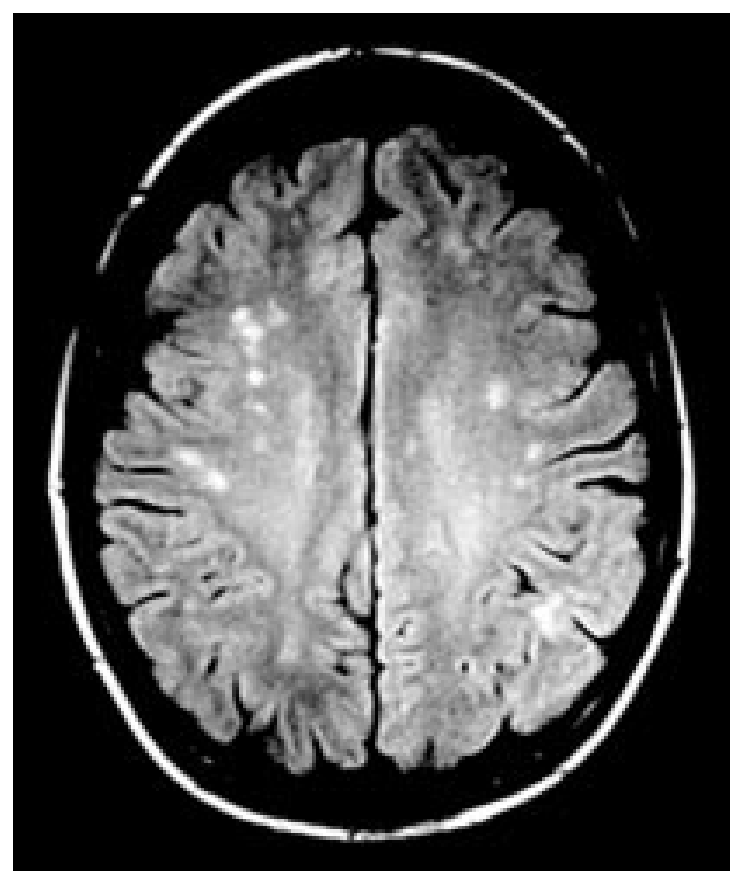

Figura 6. Imagen axial FLAIR. Nótese la presencia de pequeñas lesiones hiperintensas en la sustancia blanca bihemisférica, con una disposición más 0 menos simétrica (caso 8).

sustancia blanca subcortical no tenían deterioro cognitivo y un caso que tenía lesiones aisladas mostró deterioro cognitivo.

\section{Discusión}

Los hallazgos de este estudio permiten afirmar que más de $80 \%$ de los casos de TSP/HAM tienen alteraciones radiológicamente visibles en la médula y en el cerebro. El compromiso de la médula espinal se manifiesta en atrofia en los segmentos dorsales, sin alteración en la intensidad de señal en las secuencias T2, ni áreas de captación de gadolinio intra o perimedulares. La degeneración axomielínica se expresa en la pérdida de fibras, que van disminuyendo el volumen de la médula, sin mostrar patrón inflamatorio en los exámenes neurorradiológicos. Los mismos factores patogénicos parecen afectar ciertas áreas de la sustancia blanca subcortical ${ }^{19}$.

La atrofia de la médula espinal ha sido comunicada mayoritariamente en estudios histológi- 


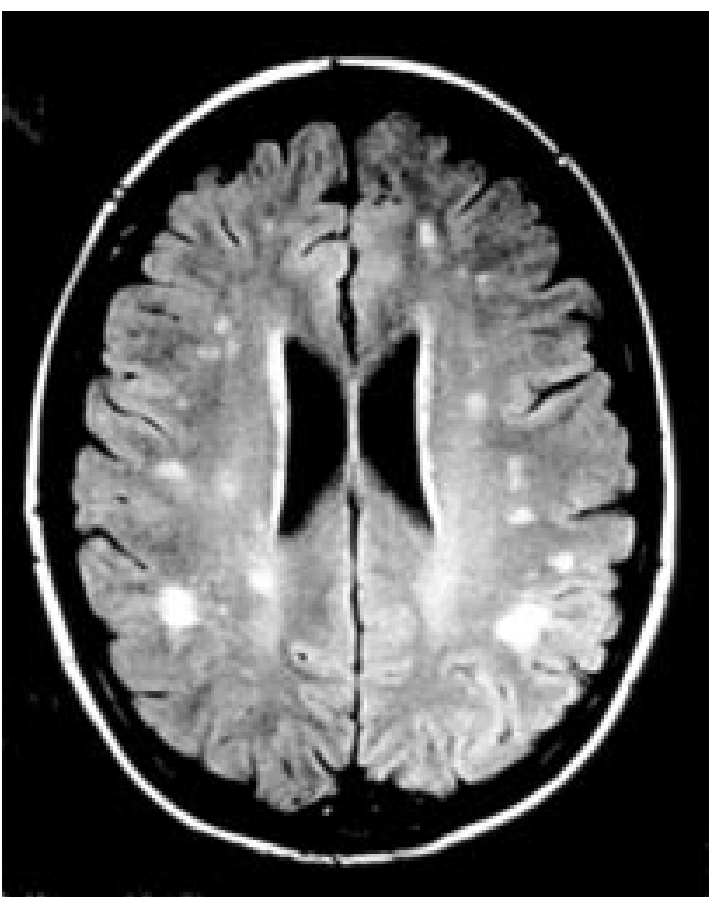

Figura 7. Imagen axial FLAIR que pone de manifiesto múltiples lesiones hiperintensas en la sustancia blanca subcortical bihemisférica, con una cierta distribución «arciforme»(caso 27).

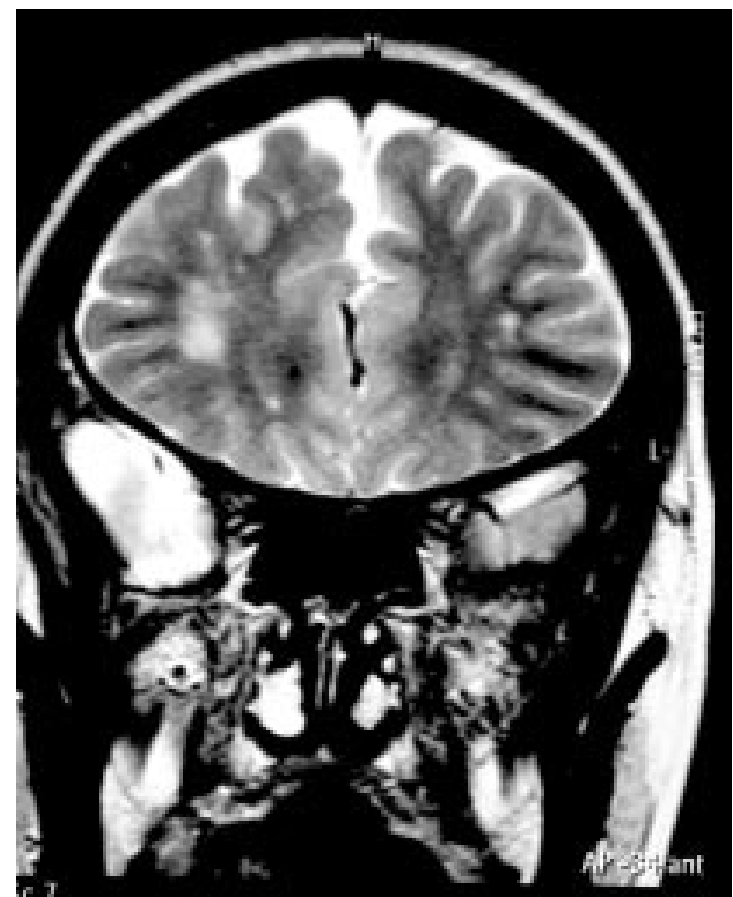

Figura 8. Imagen coronal T2. Nótese la existencia de una lesión hiperintensa confluente subcortical frontal derecha en un paciente con TSP/HAM de larga data y deterioro cognitivo (caso 22).

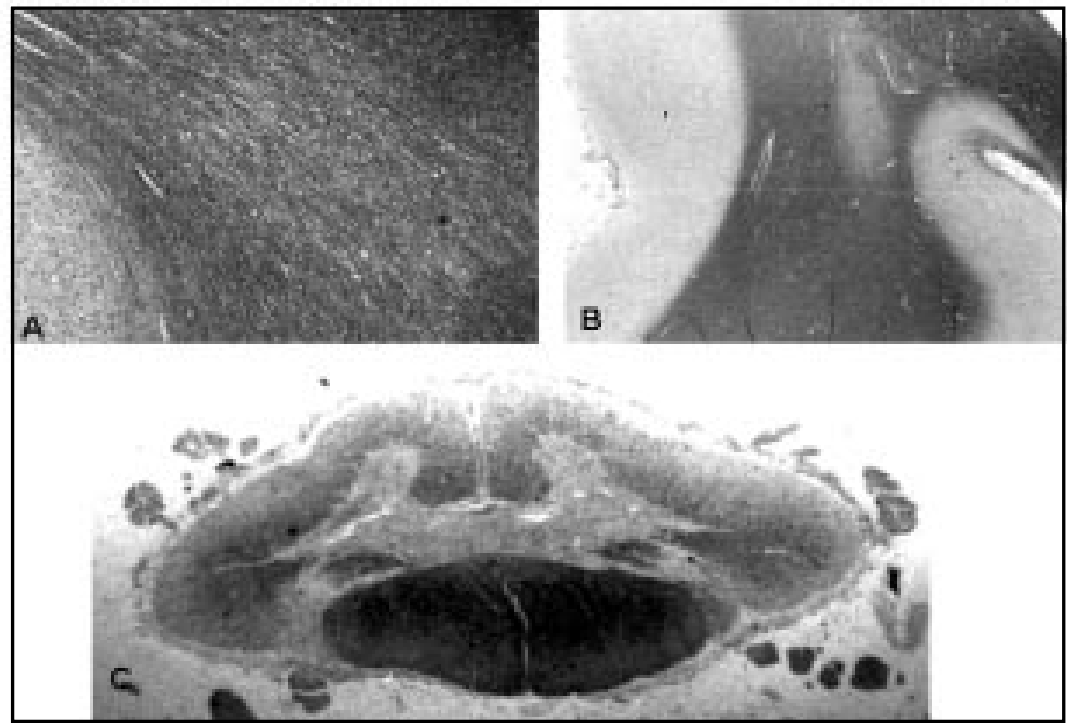

Figura 9. A) Area frontal subcortical desmielinizada, sin reacción inflamatoria ni cambios vasculares, concordante con la RM (LFB-hematoxilina x 200). B) Area frontal, lesión desmielinizante subcortical (LFBHematoxilina x 100). C) Médula dorsal: aplanamiento ántero-posterior y aspecto triangular, por pérdida de fibras en los cordones laterales (LFB-Hematoxilina x 4) (caso 2). 
$\cos ^{5-7,9,13}$ y en algunos análisis clínicos con resonancia magnética ${ }^{10,11,14}$. Sin embargo, lo original de esta presentación es la relación significativa entre el grado de atrofia de la médula espinal y la funcionalidad motora $(\mathrm{P}>0,01)$, así como el valor que puede revestir la atrofia del cono en el pronóstico de los trastornos esfinterianos y de potencia sexual en estos enfermos. Además, la correlación encontrada entre lesiones de la sustancia blanca subcortical y daño cognitivo ( $p>0,01$ ).

La atrofia de la médula espinal en la región dorsal y la disminución del volumen del cono en los pacientes con TSP/HAM, serían la expresión morfológica de una pérdida crónica de fibras mielínicas en la vía motora central. Nunca se han demostrado lesiones de la sustancia gris que justifiquen una reducción del diámetro de la médula espinal, y que se asocie a un cambio de forma (triangular) de los segmentos dorsales por la disminución del número de fibras de los cordones laterales (corticoespinales), así como por la conservación de las fibras del cordón posterior ${ }^{5}$.

En los dos pacientes en que se observó atrofia de la médula cervical, no se comprobó cambios en la motilidad, ni pérdida de la eficiencia de sus extremidades superiores. Esta aparente contradicción entre cambio estructural y daño funcional, confirma en estos pacientes el avance hacia cervical de la degeneración de las fibras cóntico-espinales que inervan las extremidades inferiores (dying-back central) después de una larga evolución de la enfermedad. El tiempo de evolución tiene una relación significativa con la disminución en el calibre de la médula ( $p>0,01)$.

Este estudio, además de caracterizar los hallazgos de la resonancia magnética en el encéfalo y en la médula espinal de pacientes con TSP/HAM, nos ha permitido establecer una relación entre el grado funcional y la severidad de la atrofia medular. El $87 \%$ de los pacientes que tienen atrofia moderada 0 severa de la médula, es coincidente con aquellos que muestran las mayores alteraciones funcionales (100\% de los pacientes sin marcha y $66,6 \%$ de aquellos con necesidad de apoyo para caminar) ( $p$ $>0,01$ ). Por el contrario, la mayoría de los casos con médula espinal normal o con leve atrofia, presentan todavía una marcha independiente. Así mismo, la atrofia del cono medular es concordante con $100 \%$ de los pacientes que presentaban incontinencia vesical e impotencia sexual y con $50 \%$ de los enfermos que padecen urgencia miccional.
El 76,6\% de los enfermos que tienen lesiones cerebrales con un spatrón confluente» de la sustancia blanca subcortical, son coincidentes con aquellos pacientes que presentan cambios intelectuales, definiendo el origen de su daño cognitivo como subcortical. Por otra parte, Ogata et $\mathrm{al}^{9} \mathrm{y}$ Cartier et $\mathrm{al}^{15}$, describieron casos de TSP/HAM con lesiones de la sustancia blanca coincidentes con estas formas de demencia subcortical. Asimismo, estudios de potenciales cognitivos (cognitive event-related potentials) y de RM demuestran que las alteraciones observadas en los potenciales evocados cognitivos, son coincidentes con lesiones de la sustancia blanca subcortical ${ }^{16,17}$.

Es habitual que lesiones de aspecto semejante a las observadas en los TSP/HAM, en la substancia blanca subcortical, hayan sido interpretadas como desmielinizantes (leucoencefalopatías) o de origen vascular ${ }^{10,11,18,19}$. Sin embargo, este compromiso de la substancia blanca cerebral debiera ser coherente con las causas que lesionan la médula y el cerebro simultáneamente. La presencia del HTLV-I como el factor etiológico y patogénico del compromiso de los axones largos del haz córtico-espinal, hace presumir un efecto semejante en el cerebro. La degeneración axomielínica de la médula es selectiva para determinado tipo de fibras motoras y sensitivas, con preservación de otras y de las neuronas. De hecho, en el TSP/HAM el daño cerebral también parece específico, porque respeta el cuerpo calloso, la sustancia blanca profunda periventricular y las fibras en U. Límite que no tienen las lesiones desmielinizantes y tampoco los fenómenos inflamatorios o vasculares.

Las lesiones de la sustancia blanca subcortical en el TSP/HAM son predominantemente anteriores, apuntando a las conexiones fronto-nucleares, aunque en el caso de Godoy et al, describieron lesiones periventriculares parecidas a las de la esclerosis múltiple ${ }^{18}$. En nuestra serie, ninguno de los pacientes presentó este aspecto, tampoco en otras series ${ }^{10,11}$.

Finalmente, la atrofia de la médula espinal y las lesiones subcorticales cerebrales asociadas a un cuadro clínico compatible, permiten establecer el diagnóstico diferencial entre TSP/HAM y otras patologías que cursan con paraparesia espástica como esclerosis múltiple, mielitis, lesiones isquémicas de cualquier origen, esclerosis lateral primaria y paraplejia espástica familiar. 


\section{REFERENCIAS}

1. Gessain A, Barin E, Vernant JC, Maurs L, Calender A, DE THÉ G ET AL. Antibodies to human Tlymphotropic virus type 1 in patients with tropical spastic paraparesis. Lancet 1985: 407-11.

2. Roman G, Osame M. Identity of HTLV-1 associated tropical spastic paraparesis and HTLV-1 associated myelopathy. Lancet 1988; I: 651.

3. Cartier L, Araya F, Castillo JL, Gormaz A, Tajima K. Progresive Spastic Paraparesis Associated with Human $t$ cell leukemia virus type I (HTLV-I). Inter Medicine 1992; 31: 1257-61.

4. Akizuki S, Nakazato O, Higuchi K, Tanake $\mathrm{K}$, Setoguchi N, Yoshida $S$ et al. Necropsy findings in HTLV-1 myelopathy. Lancet 1987; I: 156-7.

5. Cartier L, Cea JG, Vergara C, Araya F, Born P. Clinical and neuropathological study of six patients with spastic paraparesis associated with HTLV-I: An axomyelinic degeneration of the central nervous system. J Neuropathol Exp Neurol 1997; 56: 403-13.

6. Kobayashi I, Оta K, Yамamoto $\mathrm{K}$, MuRaKamin $\mathrm{H}$, NURUYAMA S, KasAZIMA T ET AL. An autopsy case of HTLV1 associated myelopathy. Neurol Med 1989; 30: 409-11.

7. IWASSAKI Y. Pathology of chronic myelopathy associated with HTLV-I infection. J Neurol Sci 1990; 96: 103-23.

8. Umehara F, Izumo S, Nakagawa M, RonquiLO AT, TaKaHASHI, Matsumoro K et al. Immunocytochemical analysis of the cellular infiltrate in the spinal cord lesions in HTLV-I associated myelophaty. J Neuropath Exp Neurol 1993; 52: 424-30.

9. Ogata A, Nagashima R, Tashino K. MRI pathological correlate of brain lesions in a necropsy case of HTLV-I associated myelopathy. J Neurol Neurosurg Psychiatry 1993; 56: 194-6.

10. Kira J, Fujihara K, Itoyama Y, Goto I, Hasuo K. Leukoencephalopathy in HTLV-1 myelopathy/ tropical spastic paraparesis: MRI analysis and a two year follow up study after corticosteroid therapy. J Neurol Scien 1991; 106: 41-9.
11. Ferraz AC, Gabbai AA, Abdala N, Gomes NogueiRA R. Ressonancia magnética na mieolopatia associado ao HTLV-I. Leucoencefalopatia e atrofia medular. Arq Neuropsiquiatria 1997; 5: 728-36.

12. Cartier L, Gormaz A. Demencia subcortical en paraparesia espástica tropical asociada a HTLV-I: Estudio de 43 casos. Rev Méd Chile 1999; 127: 444-50.

13. Bhigjee AI, Whilw CA, Wachman W. HTLV-1 associated myelopahty: clinicopathologic correlation with localization of pro-virus to the spinal cord. Neurology 1999; 41: 1990-2.

14. Nakagawa M, Izumo S, Ijichi S, Kubota $H$, Animura K, KaWABata M ET aL. HTLV-I associated myelopathy: Analysis of 213 patients based on clinical features and laboratory findings. J Neurovirology 1995; 1: 50-61.

15. Cartier L, Gormaz A, Kieinsteuber K, Ramírez E, GalENo $H$. Nueva forma de demencia subcortical: Encefalopatía por infección del virus linfotrópico humano (HTLV-I). Caso clínico. Rev Méd Chile 1997; 125: 209-13.

16. Fukushima T, Ikeda T, Uyama E. Cognitive eventrelated potentials and brain magnetic resonance imaging in HTLV-I associated myelopathy (HAM). J Neurol Sci 1994; 126: 30-9.

17. Castillo JL, Camposano S, Cartier L, Gormaz A, ACEVEDO F. Event related potentials in progresive spastic HTLV-I positive and negativa paraparesis. Rev Chil Neuropsiquiat 1995; 33: 45-50.

18. Godoy AJ, Kira J, Hasuo K, Goto I. Characterization of cerebral white matter lesions of HTLV-I associated myelopathy/tropical spastic paraparesis in comparison with multiple sclerosis and collagen-vasculitis: a semiquantitative MRI study. J Neurol Sci 1995; 133 (1-2): 102-11.

19. Kira J, Mimato S, Itoyama Y, Goto I, Kato M, Hasuo K. Leukoencephalopathy in HTLV-I associated myelopathy: MRI and EEG data. J Neurol Sci 1988; 87: 221-32.

Agradecimientos:

Los autores agradecen la valiosa colaboración de Clínica Alemana en la realización de los estudios de resonancia magnética. 